\title{
The Influence of Magnetic Turbulence on the Energetic Particle Transport Upstream of Shock Waves
}

\author{
Silvia Perri ${ }^{1, *}$, Giuseppe Prete ${ }^{1}$, Francesco Malara ${ }^{1}$, Francesco Pucci ${ }^{2,3}$ and Gaetano Zimbardo ${ }^{1}$ \\ 1 Dipartimento di Fisica, Universitá della Calabria, 87036 Rende, Italy; giuseppe.prete@unical.it (G.P.); \\ francesco.malara@fis.unical.it (F.M.); gaetano.zimbardo@fis.unical.it (G.Z.) \\ 2 Center for Mathematical Plasma Astophysics, Departement Wiskunde, KU Leuven, Universiteit Leuven, \\ 3001 Leuven, Belgium; francesco.pucci@kuleuven.be \\ 3 Istituto per la Scienza e Tecnologia dei Plasmi, Consiglio Nazionale delle Ricerche, 70126 Bari, Italy \\ * Correspondence: silvia.perri@fis.unical.it
}

check for updates

Citation: Perri, S.; Prete, G.; Malara,

F.; Pucci, F.; Zimbardo, G. The

Influence of Magnetic Turbulence on the Energetic Particle Transport

Upstream of Shock Waves.

Atmosphere 2021, 12, 508. https://

doi.org/10.3390/atmos12040508

Academic Editor: Claude Cambon

Received: 5 March 2021

Accepted: 13 April 2021

Published: 17 April 2021

Publisher's Note: MDPI stays neutral with regard to jurisdictional claims in published maps and institutional affiliations.

Copyright: (C) 2021 by the authors Licensee MDPI, Basel, Switzerland. This article is an open access article distributed under the terms and conditions of the Creative Commons Attribution (CC BY) license (https:/ / creativecommons.org/licenses/by/ $4.0 /)$.

\begin{abstract}
Energetic particles are ubiquitous in the interplanetary space and their transport properties are strongly influenced by the interaction with magnetic field fluctuations. Numerical experiments have shown that transport in both the parallel and perpendicular directions with respect to the background magnetic field is deeply affected by magnetic turbulence spectral properties. Recently, making use of a numerical model with three dimensional isotropic turbulence, the influence of turbulence intermittency and magnetic fluctuations on the energetic particle transport was investigated in the solar wind context. Stimulated by this previous theoretical work, here we analyze the parallel transport of supra-thermal particles upstream of interplanetary shock waves by using in situ particle flux measurements; the aim was to relate particle transport properties to the degree of intermittency of the magnetic field fluctuations and to their relative amplitude at the energetic particle resonant scale measured in the same regions. We selected five quasi-perpendicular and five quasi-parallel shock crossings by the ACE satellite. The analysis clearly shows a tendency to find parallel superdiffusive transport at quasi-perpendicular shocks, with a significantly higher level of the energetic particle fluxes than those observed in the quasi-parallel shocks. Furthermore, the occurrence of anomalous parallel transport is only weakly related to the presence of magnetic field intermittency.
\end{abstract}

Keywords: interplanetary shocks; turbulence; energetic particles

\section{Introduction}

Understanding the transport properties of energetic particles in space and astrophysical plasmas is crucial for assessing particle acceleration and propagation. In addition to normal diffusion, in recent decades anomalous transport regimes, characterized by a nonlinear time growth of the particle mean square displacement, i.e., $\left\langle\Delta r^{2}\right\rangle \sim t^{\alpha}$, have been found in many physical systems [1-3]. In particular, it has been well assessed how particle transport properties have a large influence on the efficiency of acceleration at shock waves. Indeed, in the case of normal diffusion, the theory of diffusive shock acceleration (DSA) predicts an exponential decay of the upstream energetic particle fluxes with distance from the shock, assuming balance between advection and particle diffusion and a spatially independent diffusion coefficent (e.g., [4]). Instead, in the case of superdiffusion, i.e., $\left\langle\Delta r^{2}\right\rangle \sim t^{\alpha}$ with $\alpha>1$, Perri and Zimbardo [5,6] starting from a Lévy walk model characterized by a power-law particle propagator far from the source/shock, have predicted that the far upstream energetic particle fluxes decay as a power-law $J \propto t^{-\beta}$, and that the exponent of superdiffusion is directly related to the slope of the power-law of the particle fluxes, $\alpha=2-\beta$. For energetic particles accelerated at collisionless shocks, superdiffusive transport has been deduced from the analysis of energetic particle time profiles upstream of heliospheric shocks [5-11], from the upstream precursor of supernova 
remnant shocks detected in the X-rays [12,13], from the galactic cosmic ray spectra [14], from the discrepancy between the radio-derived and X-ray-derived Mach numbers at the galaxy cluster merger shocks [15,16], and from self-consistent particle-in-cell simulations of protons accelerated at shocks [17].

However, the physical mechanisms at the basis of those anomalous transport behaviours are to date not well understood. It is well known that magnetic turbulence affects particle propagation in magnetized plasmas, with transport regimes being different in the directions parallel and perpendicular to the average magnetic field [18-25] (for a comprehensive review about how turbulence models affect energetic particle perpendicular transport and about the existing theories for describing the field line random walk that controls perpendicular transport). In particular, magnetic field anisotropy strongly influences the transport regimes in the parallel and perpendicular directions. It was actually found that there is evidence of parallel superdiffusive transport and perpendicular subdiffusion in quasi-slab static turbulence [26,27]. On the other hand, Hussein and Shalchi [28] by using a dynamical slab $/ 2 \mathrm{D}$ turbulence with a magnetic field fluctuation amplitude $\delta B / B=0.5$ could recover normal diffusion with energetic particle mean free paths very close to the so-called Palmer consensus [29]. In the context of the interstellar medium, turbulence tends to be anisotropic at small scales with sharp variations perpendicular to the mean magnetic field, leading to an inefficient particle scattering [30]. This problem has been extensively studied, showing that the presence of complex magnetic fields in molecular clouds leads to frequent cosmic rays' magnetic reflections that reduce cosmic ray diffusion within the Galactic disk. Recently, implementing a 3D isotropic model of static magnetic field turbulence [31] with adjustable turbulent spectral extension, fluctuation amplitude, and the degree of scale-dependent non Gaussianity, also called magnetic intermittency [32], the transport of energetic particles in the direction parallel and perpendicular to the mean field has been investigated $[33,34]$. Asymptotically, particles reach parallel and perpendicular diffusive regime (perpendicular subdiffusion is reached in case of very low magnetic field fluctuations). However, the transient (ballistic) phase, that is the time needed by the system to reach diffusion and that requires a sufficient number of particle scatterings, can last for a variable running time depending on the values of the parameters quoted above. The value of the transient time in [33] is found to be much larger than the particle scattering time (see Equation (1) below). In particular, the onset of the parallel diffusive phase starts earlier (in terms of the running times of the $3 \mathrm{D}$ isotropic turbulence simulation) when the spectral extension approaches the Larmor scale and for a $\delta B / B_{0} \sim 1$. Intermittency only weakly affects the duration of the transient phase in the parallel direction, being the onset of the diffusive propagation a bit delayed in the presence of intermittency with respect to the presence of a Gaussian fluctuation field. This is in full agreement with the cosmic-ray diffusion studied in a test-particle simulation in 3D turbulence by [33] (see their Figure 5). Pucci et al. [33] also found that intermittency has no particular influence on the transport of particles at different energies [34]. Although energetic particles perform parallel diffusion for long times, Pucci et al. [33] found that the distribution of the particles' scattering times in pitch angle (computed by following the particles' trajectories and picking-up large pitch angle variations) does not peak at a the quasi-linear prediction:

$$
\tau_{Q L T} \propto \Omega_{p}^{-1}\left(\frac{B_{0}}{\delta B}\right)^{2}\left(\frac{\ell_{c}}{\rho_{p}}\right)^{2 / 3},
$$

where $\Omega_{p}$ is the proton gyrofrequency divided by $2 \pi, \ell_{c}$ is the correlation length of turbulence (typically in the solar wind at $1 \mathrm{AU} \ell_{c} \sim 5 \times 10^{6}$ ), and $\rho_{p}$ is the particle Larmor radius [35]. In other words, once the ratio $\ell_{c} / \rho_{p}$ is fixed, the particle scattering time predicted by the quasi-linear theory (QLT) should depend on the magnetic fluctuation amplitude relative to the background field. However, numerical simulations show that the distribution of the scattering time of energetic particles broadens around the quasi-linear approximation over about five orders of magnitude [33,34], suggesting a scale-free nature of the pitch angle scattering times. Such a broadening is augmented in the presence of 
$\delta B / B_{0} \ll 1$ and high intermittency. Ref. [36] have rigorously shown how a power-law distribution of particle scattering times is the basis of an anomalous pitch angle scattering that leads to parallel spatial superdiffusion.

Stimulated from the above numerical results, we explored how the magnetic field and turbulence properties upstream of interplanetary shock waves can affect the energetic particle transport, using the in situ observations of five quasi-perpendicular and five quasiparallel shock crossings by the Advanced Composition Explorer (ACE) spacecraft at 1 AU. We analyzed in synergy the high-resolution magnetic field, plasma, and energetic particle measurements.

\section{Shock Crossings by ACE}

Events were selected according to the shock geometry, computing the angle $\theta_{B n}$ between the normal to the shock front and the mean magnetic field direction. In order to derive the normal direction, we applied the minimum variance analysis [37] to $5 \mathrm{~min}$ averaged ACE/MAG data over a 30 min interval upstream of the shock. However, when the magnetic field was found to be highly fluctuating, we compared our results with the ones in the literature [38] and on the ACE shock list (http:/ / www.ssg.sr.unh.edu/mag/ ace/ACElists/obs_list.html\#2000 (accessed on 16 April 2021)). Two examples are shown in Figure 1, where the left panel is a quasi-parallel shock crossing, while the right panel is a quasi-perpendicular one. The vertical dashed lines indicate the shock times. Other shock crossings analyzed are listed in Table 1, where all the relevant parameters are also reported (see Table 1's caption). Following the methodology described in Perri and Zimbardo [5,6] we analyzed the energetic particle fluxes upstream of the shock front over a region that goes from about $-200 \mathrm{~min}$ to $-10 \mathrm{~min}$ from the shock crossing time (this can slightly vary from event to event), assuming the shock at $t=0$. This allows us to infer the particle transport properties upstream from the discontinuity by analyzing the particle time profiles. For the two shock crossings in Figure 1, we then plotted the energetic particle fluxes in $\log -\log$ axes in Figure 2: for the quasi-parallel interval (left panel), the ion fluxes, in all the energy channels considered, are well fitted by an exponential decay $J(t) \propto \exp (-t / T)$ (black dashed lines), suggesting diffusive transport far upstream of the shock, where $T$ is the exponential time rate that is related to the particle diffusion length upstream and to the shock speed, $T=L_{\mathrm{up}} / V_{\mathrm{sh}}$ [39]. According to the best fit values of $T$ reported in the left panel of Figure 2, the energetic particle transport properties are weakly energy dependent. On the other hand, the fluxes of ions accelerated at the quasi-perpendicular event in Figure 1 exhibit a power-law decay far upstream (black dashed lines), with a slope $\beta<1$, which is consistent with a superdiffusive transport (i.e., $\left\langle\Delta r^{2}\right\rangle \propto t^{\alpha}=t^{2-\beta}$ ) with $\alpha>1[5,6]$. In this case, the degree of superdiffusion, as deduced from the $\beta$ values reported in the right panel of Figure 2, weakly depends on the particle energies. Furthermore, it is worth noting that the quasi-parallel shock on 17 June 2011 is a less efficient accelerator with respect to the quasi-perpendicular shock on 11 February 2011, although the Alfvénic Mach number $M_{A}$ (see Table 1) does not differ dramatically between the two events. $M_{A}$ has been computed upstream by averaging plasma and magnetic field measurements over a 30 min time window before the shock crossing. Actually, as can be deduced from Table 1, the flux intensity at the shock $J^{*}$ tends to be lower of about one or two orders of magnitude for the quasi-parallel events. There is only an exception for the 17 July 2002 crossing, whose ion fluxes upstream are very flat over several energy channels and abruptly decrease by one order of magnitude at $\sim 70$ min upstream of the shock front. Such a sharp decreases seems to be due to a rapid increase towards $90^{\circ}$ of the angle between the mean field direction and the radial component of $\mathbf{B}$, namely that the spacecraft tends to become disconnected from the shock.

These data suggest a more efficient acceleration at quasi-perpendicular shocks, at least for those ranges of magnetic field and plasma parameters, than at quasi-parallel shocks, with a weak dependence on the Alfvénic Mach number. This evidence can probably find an explanation in the pre-acceleration process from the thermal bath to suprathermal energies. 
Such a pre-acceleration process can be due to shock drift and/or shock surfing acceleration, which tend to be more effective at quasi-perpendicular shocks. Furthermore, looking at the intervals studied here, it is possible to note that the five quasi-perpendicular shock crossings show energetic particle fluxes upstream of the shock that are all well reproduced by a power-law time decay with slopes $\beta \in[0.2,0.4]$ corresponding to superdiffusion (see Table 1). On the contrary, the quasi-parallel crossings tend to exhibit energetic ion fluxes that are almost flat far upstream or have an exponential time decay, the latter corresponding to normal diffusion.

Table 1. Parameters of the ACE shock crossings analyzed: date of the events; time of the shock in UT; shock geometry; Alfvénic Mach number; the exponent of superdiffusion; the maximum value of the kurtosis at the Larmor scale of $100 \mathrm{keV}$ protons; the fluctuation amplitude calculated at the scale of $100 \mathrm{keV}$ protons; and the flux of the $100 \mathrm{keV}$ protons at the shock.

\begin{tabular}{|c|c|c|c|c|c|c|c|}
\hline Date & Time (UT) & $\theta_{B n}\left(^{\circ}\right)$ & $M_{A}$ & $\alpha$ & $K_{\max }(\tau)$ & $\delta B / B_{0}$ & $J^{*}\left(\mathrm{~cm}^{-2} / \mathrm{s}^{*} \mathrm{MeV}^{*} \mathrm{sr}\right)$ \\
\hline 27 January 2000 & $14: 00$ & $23 \pm 15$ & $1.6 \pm 0.4$ & ND & $8.0 \pm 0.7$ & $0.04 \pm 0.02$ & 171.6 \\
\hline 11 February 2011 & $23: 18$ & $85.4 \pm 8.8$ & $3.6 \pm 0.5$ & $1.69 \pm 0.01$ & $15.0 \pm 0.9$ & $0.09 \pm 0.03$ & $4.9 \times 10^{5}$ \\
\hline 23 June 2000 & $12: 27$ & $88.7 \pm 4.5$ & $3.5 \pm 0.2$ & $1.60 \pm 0.01$ & $5.3 \pm 1.3$ & $0.09 \pm 0.04$ & $4.4 \times 10^{5}$ \\
\hline 17 August 2001 & $10: 16$ & $68.1 \pm 5.4$ & $2.7 \pm 0.8$ & $1.62 \pm 0.01$ & $6.7 \pm 1.3$ & $0.11 \pm 0.05$ & $4.1 \times 10^{5}$ \\
\hline 17 July 2002 & $15: 26$ & $4.3 \pm 8.0$ & $4.4 \pm 0.4$ & ND & $11.6 \pm 1.2$ & $0.23 \pm 0.09$ & $3.8 \times 10^{5}$ \\
\hline 11 November 2004 & $16: 43$ & $151.6 \pm 7.7$ & $1.43 \pm 0.46$ & ND & $4.4 \pm 0.7$ & $0.05 \pm 0.02$ & $2.1 \times 10^{4}$ \\
\hline 28 May 2010 & $01: 53$ & $6.0 \pm 5.1$ & $2.76 \pm 0.1$ & ND & $4.8 \pm 0.8$ & $0.2 \pm 0.1$ & 423 \\
\hline 23 August 2010 & $16: 55$ & $124 \pm 12$ & $3.9 \pm 1.1$ & $1.77 \pm 0.01$ & $5.3 \pm 0.8$ & $0.15 \pm 0.05$ & $10^{5}$ \\
\hline 17 June 2011 & 02:01 & $40 \pm 3$ & $2.7 \pm 0.1^{2}$ & ND & $4.2 \pm 1.0$ & $0.12 \pm 0.04$ & $2.8 \times 10^{4}$ \\
\hline 12 September 2014 & $15: 26$ & $99.4 \pm 3.8$ & $2.8 \pm 0.4$ & $1.797 \pm 0.003$ & $5.3 \pm 1.5$ & $0.15 \pm 0.05$ & $6.5 \times 10^{4}$ \\
\hline
\end{tabular}
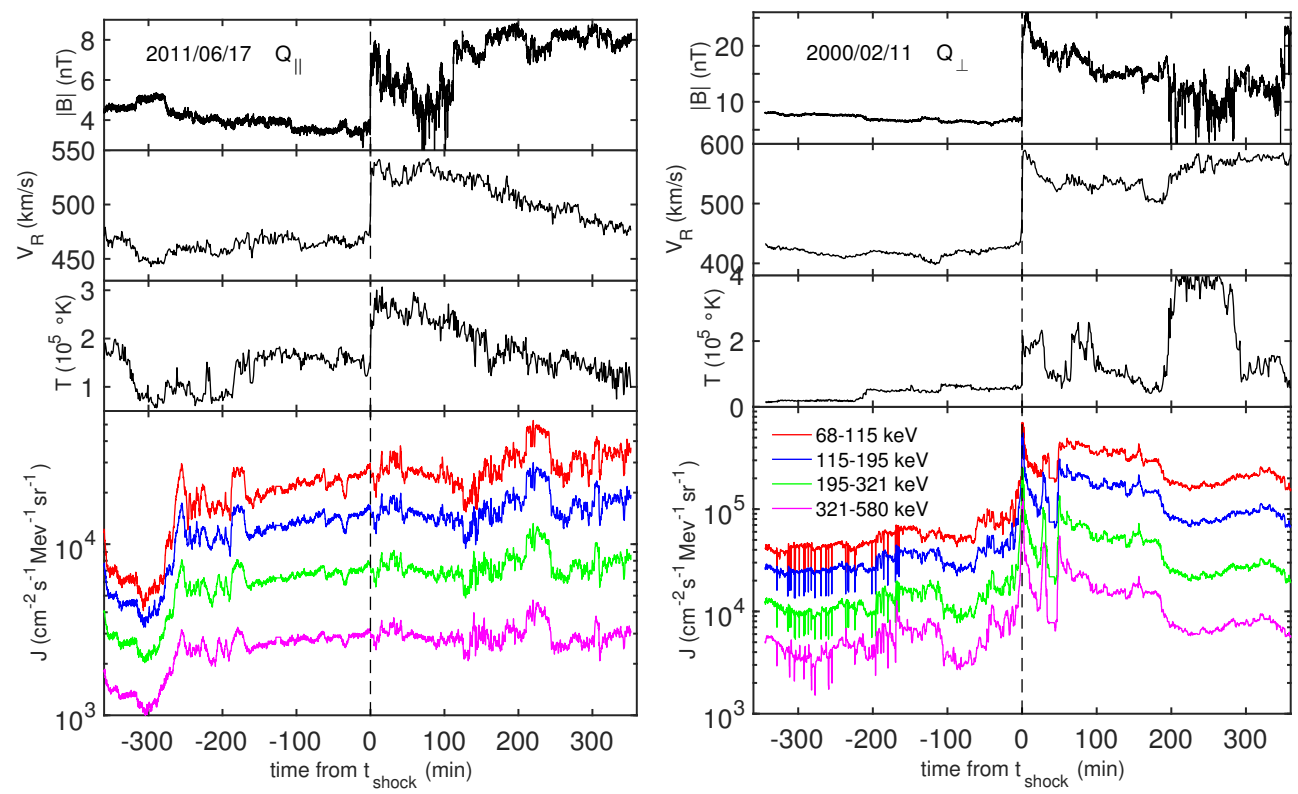

Figure 1. Two satellite shock crossings in quasi-parallel (left panels) and in quasi-perpendicular configuration (right panels). From top to bottom: the magnetic field intensity from the ACE/MAG instrument at a resolution of $1 \mathrm{vec} / \mathrm{s}$; the radial component of the solar wind bulk speed and the plasma temperature from the ACE/SWEPAM experiment at $64 \mathrm{~s}$ resolution; and the ion fluxes in four energy channels (as indicated in the legend in the right bottom panel) from the ACE/EPAM instrument at a resolution of $12 \mathrm{~s}$, as a function of the distance from the shock time (vertical dashed lines). Notice that far downstream of the 11 February 2011 event (at about 200 min from the shock), a hot (and low density) portion of the solar wind plasma occurs, also associated to larger fluctuations in $|B|$, though this is not actually related to the shock itself. 


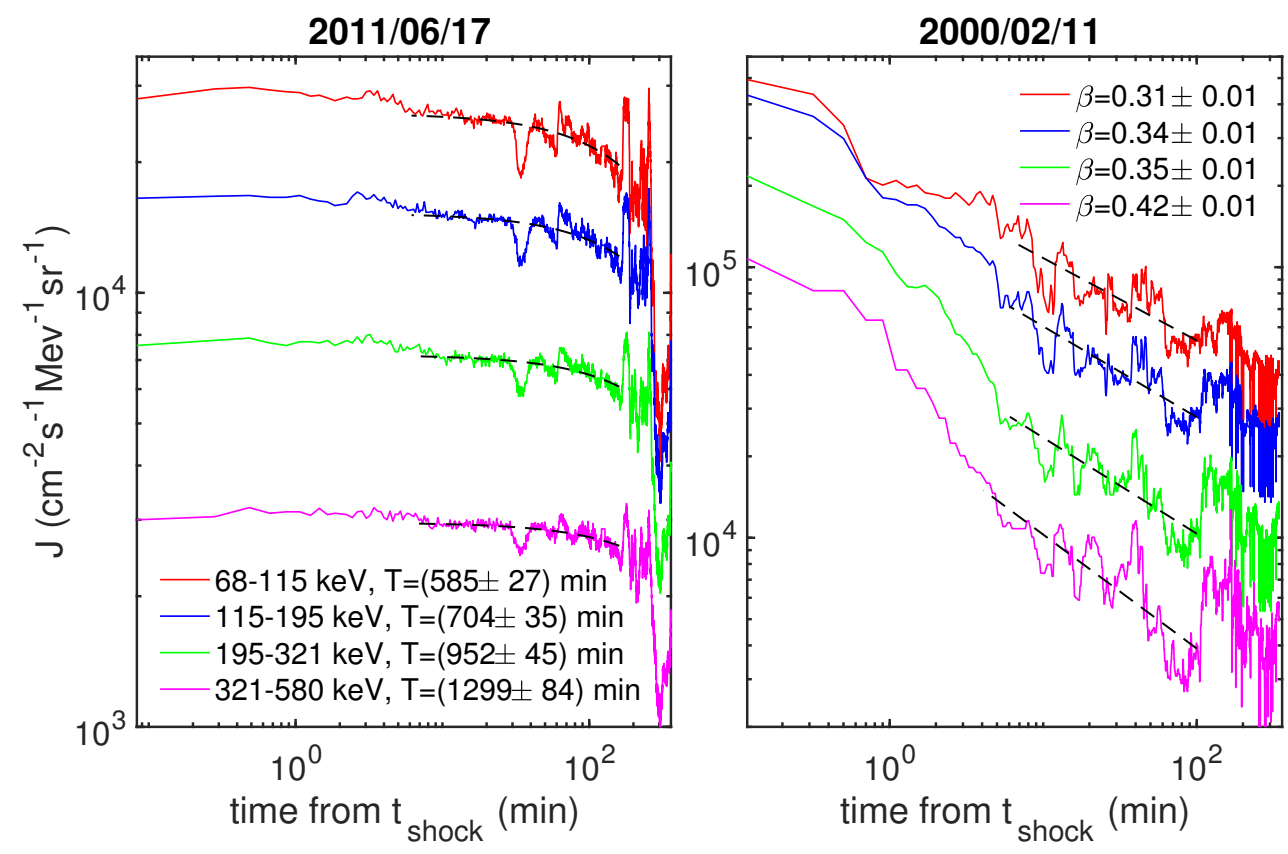

Figure 2. Plot in log-log axes of the ion energy fluxes in four different channels (as indicated in the figure legend) as a function of the distance from the shock time. For the quasi-parallel shock of the 17 June 2011 (left panel) the far upstream decay is well fitted by an exponential function $J(t) \propto \exp (-t / T)$, while in the quasi-perpendicular shock on 11 February 2011 (right panel), the ion fluxes decay as a power-law in the upstream region suggesting superdiffusive transport. The exponential and power-law best fits are reported in the panels together with their best fit parameters.

\section{The Role of Magnetic Field Intermittency on the Parallel Particle Transport}

Using in situ spacecraft data, we investigated the influence of the turbulence properties on the energetic particle transport upstream of interplanetary shocks, in order to understand whether the occurrence of the power-law time profiles for energetic particles corresponds to particular turbulence conditions. This investigation followed the numerical study in Pucci et al. [33]. Thus, we first estimated the level of intermittency of the magnetic field fluctuations in the upstream region from about -200 to $-10 \mathrm{~min}$ from the shock time for each shock crossing listed in Table 1. Magnetic field intermittency tends to modify the slope of the power spectral density of the magnetic field fluctuations [40], thus changing the power at the particle resonant scales, so it can have influence on the particle scattering. However, studying the transport of cosmic rays in three dimensional random magnetic fields, found that the presence of turbulent intermittent structures has the effect of enhancing particle diffusivity even without changing in the power spectral densities. A certain increase in energetic particle parallel diffusion with intermittency was also observed in Pucci et al. [33]. Magnetic turbulence intermittency can be quantified by using the fourth-order moment (kurtosis) of the magnetic field increments as a function of a time scale $\tau$ [32], that is:

$$
K_{i}(\tau)=\frac{\left\langle\delta B_{i}(t, \tau)\right\rangle_{t}^{4}}{\left(\left\langle\delta B_{i}(t, \tau)\right\rangle_{t}^{2}\right)^{2}}
$$

where $\delta B_{i}(t, \tau)=B_{i}(t+\tau)-B_{i}(t)$ are the magnetic field increments of the $i$-th component computed at a given time lag $\tau$ and $\langle\cdot\rangle_{t}$ represents a time average over the selected interval. $K_{i}(\tau)$ quantifies the degree of deviation of the distribution of the magnetic field increments from a Gaussian distribution, which is characterized by $K_{i}(\tau)=3$. In solar wind turbulence, as well as in turbulent non-magnetized fluids, it has been observed that $K_{i}(\tau)$ shows a higher and higher deviation from its Gaussian value, as the time scale $\tau$ decreases [41-43]. This implies that the plasma becomes increasingly non-homogeneous as the time scale becomes shorter and shorter. As reported in Pucci et al. [33], Perri 
et al. [34] energetic protons from tens of $\mathrm{keV}$ up to $1 \mathrm{MeV}$ in the presence of isotropic 3D turbulence show normal diffusion parallel to the background magnetic field. However, the distribution of the particle scattering times, computed as a change in the particle pitch angle following the particles' trajectories, exhibit a long power-law tail around the value of the particle scattering time predicted by the QLT (which, as described above, depends on the magnetic power at the particle resonant scale). This feature is seen to persist by varying the level of magnetic field intermittency and the distributions tend to slightly broaden as the level of intermittency increases. Figure 3 displays the kurtosis of the magnetic field components calculated far upstream in the radial-tangential-normal (RTN) coordinate frame as a function of the time scale for the quasi-parallel 17 June 2011 crossing (left panel) and for the quasi-perpendicular 11 February 2011 crossing (right panel). Error bars have also been estimated for each time scale as the dispersion found by running a sliding window of 100 points through the time series. The horizontal dashed lines in the panels in Figure 3 indicate the Gaussian level of the kurtosis, while the vertical solid lines the resonant time scale corresponding to the gyroradius of $100 \mathrm{keV}$ protons. Namely, $\tau^{-1}=f_{100 \mathrm{keV}}=V_{\text {up }} /\left(2 \pi \rho_{100 \mathrm{keV}}\right)$ where $V_{\text {up }}$ is the proton bulk speed in the upstream region and $\rho_{100 \mathrm{keV}}$ is the $100 \mathrm{keV}$ particle Larmor radius.

In the quasi-perpendicular crossing where parallel superdiffusion can be inferred from the ion fluxes in the right panel of Figure 2, the level of magnetic intermittency is higher, especially in the tangential direction. We can speculate that such a high intermittency level, being associated to the larger non-homogeneity of the plasma magnetic field, can induce features in the particles' trajectories, such as very long flights and very short trapping centers (i.e., the scale-free nature of the particle paths), probably caused by the clusterization of the magnetic field fluctuations. Furthermore, in case of quasi-perpendicular shocks, the spacecraft time series are roughly related, assuming the Taylor hypothesis, to wave vector components perpendicular to the mean field, while in the quasi-parallel configuration they are mostly related to the parallel wave vector component. Plots in Figure 3 agree with the very well known result that in magnetohydrodynamics (MHD), with a background magnetic field, the cascade is more efficient in transferring energy along the perpendicular wave vector components. On the other hand, we reported in Table 1 the maximum value of the kurtosis reached among the three magnetic field components at the time scale corresponding to the Larmor radius of $100 \mathrm{keV}$ particles: from the values shown, it is difficult to infer a conclusive assessment on the influence of magnetic field intermittency on the particle transport. Indeed, as we will see below, there are many ingredients that can have an effect on the energetic particle transport. Furthermore, Pucci et al. [33], Perri et al. [34] have pointed out how intermittency can only slightly broaden the distribution of the particle scattering times. We claim that the evidence of a weak correlation between parallel particle transport and turbulence intermittency is due to the fact that the former is mostly influenced by the turbulent fluctuations at the particle resonant scale, while the latter is a multi-scale process that tends to create non-homogeneity and the formation of sparse coherent structures towards smaller and smaller time/spatial scales [44]. On the other hand, it has been shown in Pucci et al. [33] that turbulence intermittency does not affect the perpendicular transport of energetic particles in isotropic turbulence. However, this aspect is out of the scope of the present work and deserves further investigation. 

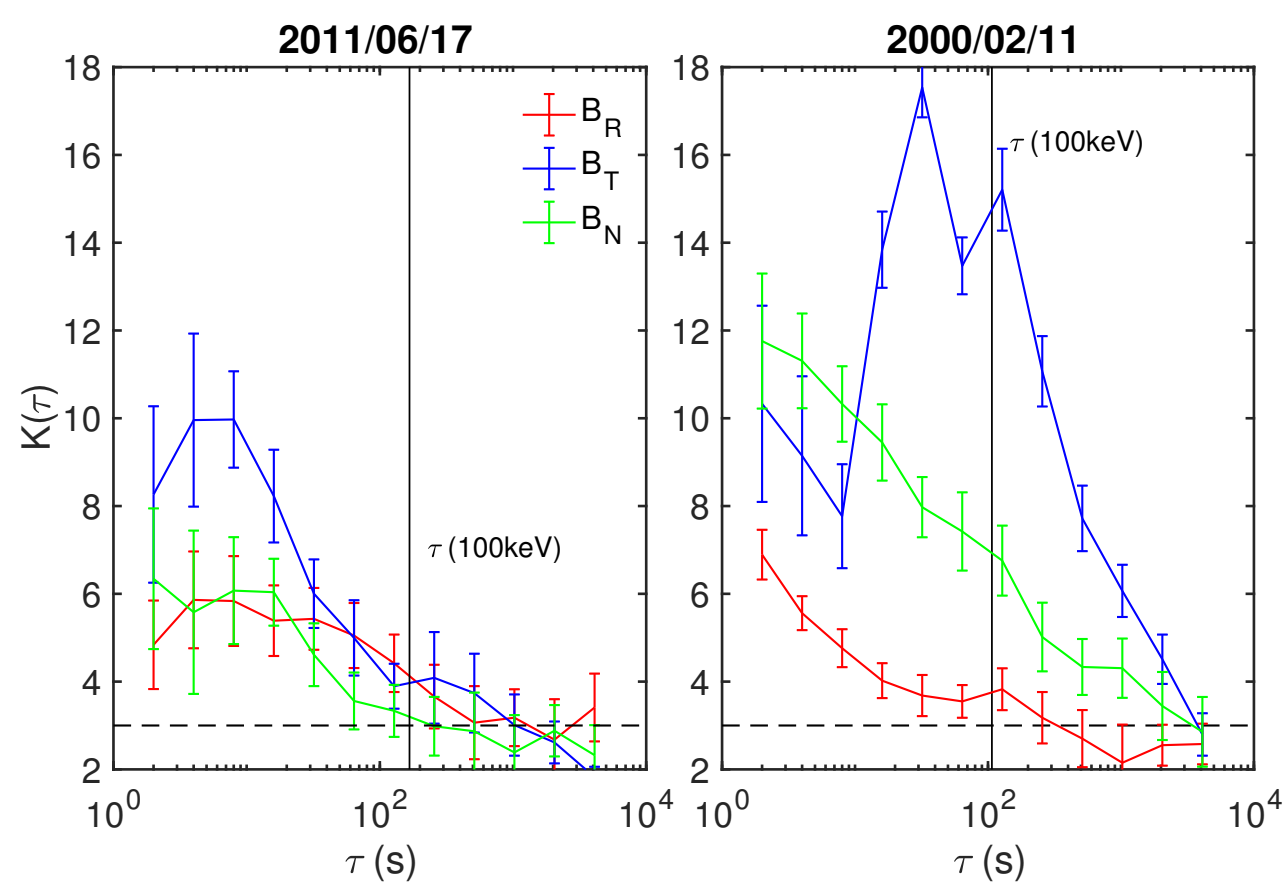

Figure 3. Kurtosis as a function of the time scale $\tau$ in the quasi-parallel shock crossing of the 17 June 2011 (left panel) and in the quasi-perpendicular shock crossing on 11 February 2011 (right panel). The Gaussian level of 3 is indicated by the horizontal dashed line and the time scale corresponding to the Larmor radius of energetic protons of $100 \mathrm{keV}$ is shown by the vertical solid line. Error bars are also reported.

\section{Particle Scattering Times}

In the list of shock crossings analyzed herein, beyond the quasi-parallel and the quasi-perpendicular configurations, there are several parameters that exhibit variations from event to event, as the Alfvénic Mach number, the mean magnetic field $B_{0}$ in the upstream region, and the level of relative fluctuations in the magnetic field (see Table 1). The $\delta B / B_{0}$ in Table 1 was computed from the variance of the magnetic field components $B_{i}$, i.e., $\sigma_{i}^{2}=\left\langle\left(B_{i}-\left\langle B_{i}\right\rangle\right)^{2}\right\rangle_{\tau}$, averaging over a time scale corresponding to the Larmor radius of $100 \mathrm{keV}$ particles (see above). Thus, the total variance was obtained as $\sigma^{2}=\sum_{i=1}^{3} \sigma_{i}^{2}$ and finally $\delta B / B_{0}=\sqrt{\sigma^{2} / B_{0}^{2}}$. Error bars were also reported and were estimated as the $\delta B / B_{0}$ dispersion using a running window upstream of time length corresponding to the $100 \mathrm{keV}$ proton gyroradius. From Table 1 , it can be seen that $\delta B / B_{0}$ is neither correlated with the shock geometry nor with the Mach number, and that $\delta B / B_{0} \ll 1$, so that we can assume it to be in the quasi-linear regime. This greatly simplifies the analysis of the particle scattering times, recovering the expression in Equation (1). In such an approximation $\tau_{Q L T} \propto \Omega_{p}^{-1}\left(\delta B / B_{0}\right)^{-2}$, namely the scattering times are directly related to the level of fluctuations at the resonant scale (apart from factors of order one). As pointed out in Perri and Zimbardo [45], $\tau_{Q L T}$ is basically related to the distribution of the magnetic field variance (i.e., on the amplitude of $\delta B / B_{0}$ at the resonant scale), so that a high intermittency in the magnetic field would lead to a broader distribution of the field variances; $\tau_{\mathrm{QLT}}$ is also directly proportional to $B_{0}$. All these dependencies need to be taken into account in order to interpret the distribution of the scattering times in each shock crossing, since those quantities tend to largely vary from event to event. Since from the analysis reported in Section 3, we did not find strong evidence of correlation between magnetic field fluctuation properties and shock geometry, we then tried to isolate different effects that can affect the distribution of the particle scattering times. Figure 4 displays the probability density functions (PDFs) of the $100 \mathrm{keV}$ scattering times $\tau_{Q L T}$ for a few events from the list in Table 1. The PDFs are divided in two groups: the first where the kurtosis is confined 
between $[4.2,5.3], B_{0}$ does not change dramatically, while $\delta B / B_{0}$ varies (left panel); and the second where $\delta B / B_{0}$ is limited between $[0.11,0.15]$, the $B_{0}$ amplitude is quite stable and the kurtosis at the resonant scale varies (right panel). This methodology should allows us to better separate the different effects. As expected, in the left panel a low level of fluctuations increases the values of the particle scattering times, as well as a larger mean magnetic field; however, the distributions do not seem to evolve towards a power-law distribution of $\tau_{Q L T}$, since the level of intermittency is pretty low. On the other hand, in the right panel in Figure 4, an evolution of the $P D F\left(\tau_{Q L T}\right)$ towards a power-law can be clearly seen in the green line, which corresponds to a quasi-perpendicular shock crossing, with high intermittency at the $100 \mathrm{keV}$ particle resonant scale and a power-law flux of energetic ions in the upstream region. The formation of a power-law distribution of scattering times can be indeed the starting point for the generation of parallel spatial superdiffusion [36,46].

We remark that the quasi-linear scattering times are rather long, reaching $\tau_{Q L T} \simeq 10^{3}-$ $10^{4} \mathrm{~s}$. While this allows for very long particle displacements $\Delta x_{\|}=v_{\|} \tau_{Q L T}$, which are an ingredient of superdiffusion, it would become difficult to explain acceleration by DSA, which requires short scattering times in order to trap energetic particles close to the shock. This problem can be solved by superdiffusive shock acceleration [47] as it encompasses a scale-free distribution of scattering times, so that short scattering times are also present [10], as can be seen in the right panel of Figure 4 .
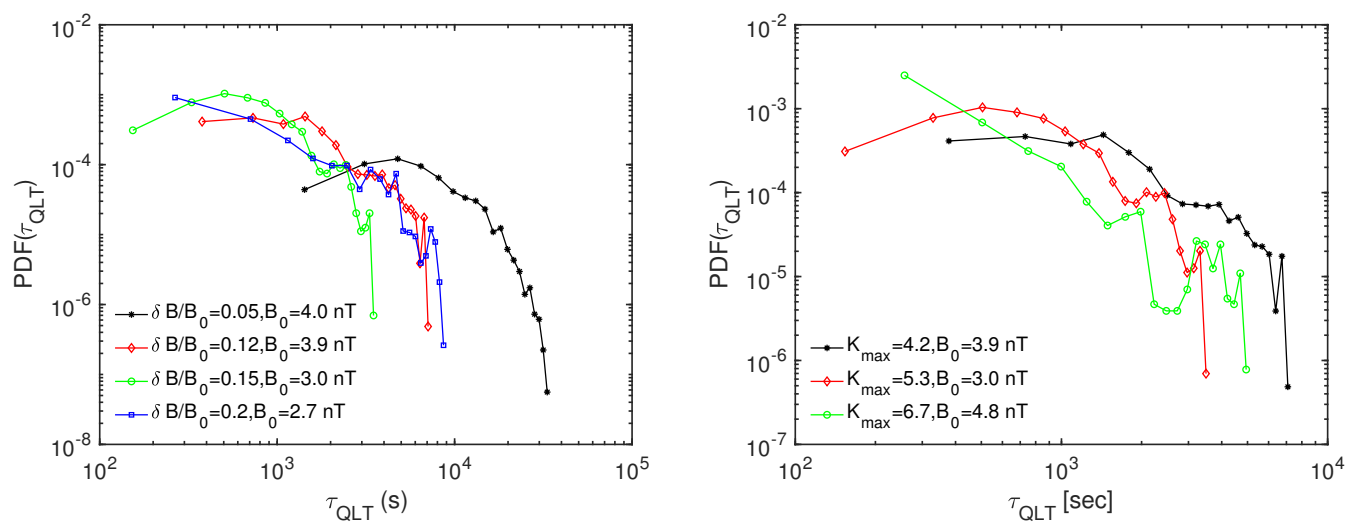

Figure 4. Probability density functions of the $100 \mathrm{keV}$ energetic particles' scattering times computed upstream of shock crossings with different levels of $\delta B / B_{0}$ and similar intermittency (left panel) and with different intermittency values but a similar $\delta B / B_{0}$ (right panel).

\section{Conclusions}

In this paper, we analyzed the magnetic field properties and the energetic particle fluxes upstream of a number of interplanetary shocks observed by the ACE spacecraft, with the goal of unraveling the relationship between magnetic turbulence properties and particle transport properties. We selected five quasi-parallel and five quasi-perpendicular shock crossings. Analyzing the upstream energetic particle fluxes, we found that transport can be found to be normal for quasi-parallel shocks, while it tends towards superdiffusion for quasi-perpendicular shocks. We also found that the intensity of the energetic particles is typically larger for quasi-perpendicular shocks: this varies with expectations from DSA, in particular when the case of a supernova remnant shocks is considered $[48,49]$. This finding can probably be explained by the fact that before entering the DSA Fermi mechanism, a pre-acceleration from the thermal bath to suprathermal energies is necessary-the socalled injection problem. This pre-acceleration can arguably be given by shock drift acceleration and shock surfing acceleration, mechanisms which are both more effective in the case of quasi-perpendicular shocks [50,51]. From our study, it appears that these mechanisms are able to inject a number of particles into Fermi acceleration, which is one or two orders of magnitude larger than those injected at quasi-parallel shocks. On the other hand, the energetic particle intensity at shocks can depend on other parameters like the Mach numbers, the compression ratio, and the presence of seed particles to be injected 
in the acceleration process, so that further studies are needed in order to gain a more complete picture.

For the first time, we also analyzed the relation between upstream energetic particle fluxes, turbulence intermittency, and quasi-linear pitch angle scattering times. We found that the rate of superdiffusion was only weakly related to the magnetic turbulence intermittency upstream of the shock, in agreement with the numerical results in Pucci et al. [33], although intermittency can vary the slope of the magnetic power spectral density, changing the magnetic power stored at the energetic particle resonant scales. We argue that this is probably due to the scale-dependent nature of intermittency, which in the interplanetary space tends to be higher at smaller and smaller scales towards the thermal particle gyroradius, while it is closer to the Gaussian level at MHD scales. This comes from the generation, through the turbulent cascade, of more and more localized and nonhomogeneously distributed structures from large to small spatial scales. However, the effect of magnetic intermittency on the distribution of the quasi-linear pitch angle scattering times is to modify it towards a power-law over two or more decades (in agreement with Perri et al. [34], Riordan and Pe'er [52]). This can have important implications on the energetic particle dynamics, since power-law distributions are the basic ingredient of anomalous transport [53].

Finally, we point out that a future study of each shock event will include the determination of the breakpoint in the particle flux power-law profiles, leading to the determination of the anomalous spatial diffusion coefficient and of the anomalous pitch angle scattering coefficient, making use of the methods developed in Perri and Zimbardo [10], Zimbardo and Perri [36].

Author Contributions: Conceptualization, S.P. and G.Z.; methodology, S.P.; data analysis, S.P. and G.P.; original draft preparation, S.P.; writing—review and editing, , S.P., F.M., F.P. and G.Z. All authors have read and agreed to the published version of the manuscript.

Funding: The resources and services used for this work were partly provided by the VSC (Flemish Supercomputer Centre), funded by the Research Foundation-Flanders (FWO) and the Flemish Government. We acknowledge the CINECA award under the ISCRA initiative IsC59_PDSMT for the availability of high performance computing resources and support. The work by F.P. has been supported by the Flanders Research Foundation (FWO) postdoctoral fellowship $12 \mathrm{X} 0319 \mathrm{~N}$ and by the FWO research grant 1507820 N. The research by G.P. acknowledges POR Calabria FSE/FESR 2014-2020 for financial support.

Data Availability Statement: Data are publicly available on https:/ /cdaweb.gsfc.nasa.gov/index. $\mathrm{html} /$ (accessed on 16 April 2021).

Acknowledgments: We acknowledge useful discussions with D. Trotta.

Conflicts of Interest: The authors declare no conflict of interest.

\section{References}

1. Sokolov, I.M.; Klafter, J. From diffusion to anomalous diffusion: A century after Einstein's Brownian motion. Chaos 2005, 15, 026103. [CrossRef]

2. Zimbardo, G.; Amato, E.; Bovet, A.; Effenberger, F.; Fasoli, A.; Fichtner, H.; Furno, I.; Gustafson, K.; Ricci, P.; Perri, S. Superdiffusive transport in laboratory and astrophysical plasmas. J. Plasma Phys. 2015, 81, 495810601. [CrossRef]

3. Sioulas, N.; Isliker, H.; Vlahos, L.; Koumtzis, A.; Pisokas, T. Superdiffusive stochastic Fermi acceleration in space and energy. Mon. Not. R. Astron. Soc. 2020, 491, 3860-3869. [CrossRef]

4. Drury, L.O. An introduction to the theory of diffusive shock acceleration of energetic particles in tenuous plasmas. Rep. Prog. Phys. 1983, 46, 973. [CrossRef]

5. Perri, S.; Zimbardo, G. Evidence of superdiffusive transport of electrons accelerated at interplanetary shocks. Astrophys. J. Lett. 2007, 671, L177-L180. [CrossRef]

6. Perri, S.; Zimbardo, G. Superdiffusive transport of electrons accelerated at corotating interaction regions. J. Geophys. Res. (Space Phys.) 2008, 113, A03107. [CrossRef]

7. Perri, S.; Zimbardo, G. Ion superdiffusion at the solar wind termination shock. Astrophys. J. Lett. 2009, 693, L118-L121. [CrossRef]

8. Perri, S.; Yordanova, E.; Carbone, V.; Veltri, P.; Sorriso-Valvo, L.; Bruno, R.; André, M. Magnetic turbulence in space plasmas: Scale-dependent effects of anisotropy. J. Geophys. Res. Space Phys. 2009, 114. [CrossRef] 
9. Sugiyama, T.; Shiota, D. Sign for Super-diffusive Transport of Energetic Ions Associated with a Coronal-mass-ejection-driven Interplanetary Shock. Astrophys. J. Lett. 2011, 731, L34. [CrossRef]

10. Perri, S.; Zimbardo, G. Short Acceleration Times from Superdiffusive Shock Acceleration in the Heliosphere. Astrophys. J. 2015, 815, 75. [CrossRef]

11. Perri, S.; Zimbardo, G.; Effenberger, F.; Fichtner, H. Parameter estimation of superdiffusive motion of energetic particles upstream of heliospheric shocks. Astron. Astrophys. 2015, 578, A2. [CrossRef]

12. Perri, S.; Amato, E.; Zimbardo, G. Transport of relativistic electrons at shocks in shell-type supernova remnants: diffusive and superdiffusive regimes. Astron. Astrophys. 2016, 596, A34. [CrossRef]

13. Perri, S. Superdiffusion of relativistic electrons at supernova remnant shocks. Plasma Phys. Controll. Fusion 2018, 60, 014005. [CrossRef]

14. Khiali, B.; Haino, S.; Feng, J. Anomalous Galactic Cosmic Rays in the Framework of AMS-02. Astrophys. J. 2017, 835, 229. [CrossRef]

15. Zimbardo, G.; Perri, S. Superdiffusive shock acceleration at galaxy cluster shocks. Nat. Astron. 2017, 1, 0163. [CrossRef]

16. Zimbardo, G.; Perri, S. Understanding the radio spectral indices of galaxy cluster relics by superdiffusive shock acceleration. Mon. Not. R. Astron. Soc. 2018, 478, 4922-4930. [CrossRef]

17. Trotta, D.; Burgess, D.; Prete, G.; Perri, S.; Zimbardo, G. Particle transport in hybrid PIC shock simulations: A comparison of diagnostics. Mon. Not. R. Astron. Soc. 2020, 491, 580-595. [CrossRef]

18. Jokipii, J.R. Cosmic-Ray Propagation. I. Charged Particles in a Random Magnetic Field. Astrophys. J. 1966, 146, 480. [CrossRef]

19. Matthaeus, W.; Qin, G.; Bieber, J.; Zank, G. Nonlinear collisionless perpendicular diffusion of charged particles. Astrophys. J. Lett. 2003, 590, L53. [CrossRef]

20. Webb, G.; Zank, G.; Kaghashvili, E.K.; Le Roux, J. Compound and perpendicular diffusion of cosmic rays and random walk of the field lines. I. Parallel particle transport models. Astrophys. J. 2006, 651, 211. [CrossRef]

21. Shalchi, A.; Kourakis, I. A new theory for perpendicular transport of cosmic rays. Astron. Astrophys. 2007, 470, 405-409. [CrossRef]

22. Dosch, A.; Shalchi, A. Diffusive shock acceleration at interplanetary perpendicular shock waves: Influence of the large scale structure of turbulence on the maximum particle energy. Adv. Space Res. 2010, 46, 1208-1217. [CrossRef]

23. Shalchi, A. Applicability of the Taylor-Green-Kubo formula in particle diffusion theory. Phys. Rev. E 2011, 83, 046402. [CrossRef]

24. Zimbardo, G.; Perri, S.; Pommois, P.; Veltri, P. Anomalous particle transport in the heliosphere. Adv. Space Res. 2012, 49, 1633-1642. [CrossRef]

25. Ferrand, G.; Danos, R.J.; Shalchi, A.; Safi-Harb, S.; Edmon, P.; Mendygral, P. Cosmic Ray Acceleration at Perpendicular Shocks in Supernova Remnants. Astrophys. J. 2014, 792, 133. [CrossRef]

26. Zimbardo, G.; Pommois, P.; Veltri, P. Superdiffusive and subdiffusive transport of energetic particles in solar wind anisotropic magnetic turbulence. Astrophys. J. Lett. 2006, 639, L91. [CrossRef]

27. Pommois, P.; Zimbardo, G.; Veltri, P. Anomalous, non-Gaussian transport of charged particles in anisotropic magnetic turbulence. Phys. Plasmas 2007, 14, 012311. [CrossRef]

28. Hussein, M.; Shalchi, A. Simulations of Energetic Particles Interacting with Dynamical Magnetic Turbulence. Astrophys. J. 2016, 817, 136. [CrossRef]

29. Palmer, I. Transport coefficients of low-energy cosmic rays in interplanetary space. Rev. Geophys. 1982, 20, 335-351. [CrossRef]

30. Matthaeus, W.H.; Goldstein, M.L.; Roberts, D.A. Evidence for the presence of quasi-two-dimensional nearly incompressible fluctuations in the solar wind. J. Geophys. Res 1990, 95, 20673-20683. [CrossRef]

31. Malara, F.; Di Mare, F.; Nigro, G.; Sorriso-Valvo, L. Fast algorithm for a three-dimensional synthetic model of intermittent turbulence. Phys. Rev. E 2016, 94, 053109. [CrossRef] [PubMed]

32. Frisch, U. Turbulence: The Legacy of AN Kolmogorov; Cambridge University Press: Cambridge, MA, USA, 1995.

33. Pucci, F.; Malara, F.; Perri, S.; Zimbardo, G.; Sorriso-Valvo, L.; Valentini, F. Energetic particle transport in the presence of magnetic turbulence: influence of spectral extension and intermittency. Mon. Not. R. Astron. Soc. 2016, 459, 3395-3406. [CrossRef]

34. Perri, S.; Pucci, F.; Malara, F.; Zimbardo, G. On the Power-Law Distribution of Pitch-Angle Scattering Times in Solar Wind Turbulence. Sol. Phys. 2019, 294, 34. [CrossRef]

35. Amato, E. The origin of galactic cosmic rays. Int. J. Mod. Phys. D 2014, 23, 1430013. [CrossRef]

36. Zimbardo, G.; Perri, S. Non-Markovian Pitch-angle Scattering as the Origin of Particle Superdiffusion Parallel to the Magnetic Field. Astrophys. J. 2020, 903, 105. [CrossRef]

37. Sonnerup, B.U.Ö.; Cahill, L.J. Explorer 12 observations of the magnetopause current layer. J. Geophys. Res. $1968,73,1757$. [CrossRef]

38. Yang, L.; Wang, L.; Li, G.; Wimmer-Schweingruber, R.F.; He, J.; Tu, C.; Tian, H.; Bale, S.D. Electron Acceleration by ICME-driven Shocks at 1 au. Astrophys. J. 2019, 875, 104. [CrossRef]

39. Giacalone, J. Energetic Charged Particles Associated with Strong Interplanetary Shocks. Astrophys. J. 2012, 761, 28. [CrossRef]

40. Veltri, P.; Mangeney, A. Scaling laws and intermittent structures in solar wind MHD turbulence. In Solar Wind Nine; Habbal, S.R., Esser, R., Hollweg, J.V., Isenberg, P.A., Eds.; American Institute of Physics Conference Series: Melville, NY, USA, 1999; Volume 471, pp. 543-546. 
41. Marsch, E.; Tu, C.Y. Intermittency, non-Gaussian statistics and fractal scaling of MHD fluctuations in the solar wind. Nonlinear Process. Geophys. 1997, 4, 101-124. [CrossRef]

42. Sorriso-Valvo, L.; Carbone, V.; Veltri, P.; Consolini, G.; Bruno, R. Intermittency in the solar wind turbulence through probability distribution functions of fluctuations. Geophys. Res. Lett. 1999, 26, 1801-1804. [CrossRef]

43. Bruno, R.; Carbone, V. The Solar Wind as a Turbulence Laboratory. Living Rev. Sol. Phys. 2013, 10, 2. [CrossRef]

44. Yordanova, E.; Perri, S.; Sorriso-Valvo, L.; Carbone, V. Multipoint observation of anisotropy and intermittency in solar-wind turbulence. Europhys. Lett. 2015, 110, 19001. [CrossRef]

45. Perri, S.; Zimbardo, G. Magnetic variances and pitch-angle scattering times upstream of interplanetary shocks. Astrophys. J. 2012b, 754, 8. [CrossRef]

46. Zimbardo, G.; Perri, S. From Lévy walks to superdiffusive shock acceleration. Astrophys. J. 2013, 778, 35. [CrossRef]

47. Perri, S.; Zimbardo, G. Superdiffusive shock acceleration. Astrophys. J. 2012, 750, 87. [CrossRef]

48. Reynoso, E.M.; Hughes, J.P.; Moffett, D.A. On the radio polarization signature of efficient and inefficient particle acceleration in supernova remnant sn 1006. Astron. J. 2013, 145, 104. [CrossRef]

49. Caprioli, D.; Spitkovsky, A. Simulations of Ion Acceleration at Non-relativistic Shocks. III. Particle Diffusion. Astrophys. J. 2014, 794, 47. [CrossRef]

50. Yang, Z.W.; LembèGe, B.; Lu, Q.M. Acceleration of heavy ions by perpendicular collisionless shocks: Impact of the shock front nonstationarity. J. Geophys. Res. (Space Phys.) 2011, 116, A10202. [CrossRef]

51. Yang, Z.W.; Lembège, B.; Lu, Q.M. Impact of the rippling of a perpendicular shock front on ion dynamics. J. Geophys. Res. (Space Phys.) 2012, 117, A07222. [CrossRef]

52. Riordan, J.D.; Pe'er, A. Pitch-angle Diffusion and Bohm-type Approximations in Diffusive Shock Acceleration. Astrophys. J. 2019, 873, 13. [CrossRef]

53. Klafter, J.; Blumen, A.; Shlesinger, M.F. Stochastic pathway to anomalous diffusion. Phys. Rev. A 1987, 35, 3081-3085. [CrossRef] 